\title{
Adesão à terapêutica na asma
}

\section{Adherence to therapy in asthma}

Data de receção / Received in: I 1/02/2019 Data de aceitação / Accepted for publication in: 18/03/2019

Rev Port Imunoalergologia 2020;28 (2):87-95

Marta Martins, Bárbara Cardoso, Sofia Farinha, Rute Reis, Elza Tomaz, Filipe Inácio

Hospital São Bernardo, Setúbal

\section{RESUMO}

Introdução: A adesão à terapêutica na asma brônquica é fundamental para o controlo da doença. Diversos estudos mostram que a não adesão parece ser o resultado de diferentes fatores e barreiras, associados ao doente, mas também ao prescritor. Referem-se como mais importantes os aspetos psicológicos, económicos e sociais. Na prática clínica existem poucos recursos que permitam ao médico objetivar o grau de cumprimento da sua prescrição. $O$ objetivo deste estudo foi analisar o grau de adesão à terapêutica dos doentes asmáticos seguidos em consulta de especialidade de Imunoalergologia. Métodos: Estudaram-se retrospetivamente os processos clínicos de 63 doentes asmáticos acompanhados na consulta de Imunoalergologia entre janeiro e dezembro de 2016 (T0) e de janeiro a dezembro de 2017 (TI). Através dos registos da Prescrição Electrónica de Medicamentos (PEM) foi analisado, em T0 e $\mathrm{TI}$, o número de embalagens prescritas pelo médico assistente e o número das embalagens efetivamente adquiridas nas farmácias relativamente aos seguintes fármacos: broncodilatadores (BD), corticoides inalados isolados ou em associação com broncodilatadores (OUT), anti-histamínicos orais (AH), antagonistas dos leucotrienos (LCRA), corticoides nasais $(\mathrm{CN})$ e corticoides orais $(\mathrm{CO})$. Analisaram-se ainda as seguintes variáveis demográficas e clínicas: idade, sexo, diagnóstico clínico, atopia, sensibilização alergénica e realização de imunoterapia específica (ITE). Resultados: Constatamos uma adesão geral à prescrição de 64,76\%. Os medicamentos com maior adesão foram os CO (73\%), seguidos dos LCRA e dos $\mathrm{AH}(70 \%)$. Na análise de associações entre variáveis constatou-se que os doentes que não faziam ITE apresentaram maior adesão aos inaladores (BD e OUT) $(p<0,05)$. $O$ grupo de doentes asmáticos apresentou uma associação positiva com a adesão aos LCRA $(p<0,05)$, e na análise por idades verificamos que a população infantil apresentou uma associação positiva com a adesão aos $\mathrm{AH}(\mathrm{p}<0,05)$. Conclusões: Para melhorar a adesão à terapêutica na asma importa abordar e conhecer a adesão dos doentes. $O$ estudo da adesão baseado nos sistemas informáticos de prescrição e levantamento de medicamentos nas farmácias permite aos médicos prescritores introduzir esta variável na análise do controlo da asma.

Palavras-chave: Adesão, asma, consumo de medicamentos, prescrição eletrónica. 


\section{ABSTRACT}

Background: Adherence to therapy in bronchial asthma is essential for the control of the disease. Several studies show that non-adherence seems to be the result of different factors and barriers associated with the patient, but also with the prescriber. The most important are the psychological, economic and social aspects. In clinical practice, there are few resources that allow the physician to objectify the degree of compliance of his prescription. The aim of this study was to analyze the degree of adherence to therapy in asthmatic patients followed in a Hospital Immunoallergology Department. Methods: The clinical trials of 63 asthmatic patients followed at the Immunoallergology Department from January to December of 2016 (TO) and from January to December of 2017 (TI) were retrospectively studied. The number of packs prescribed to the patients by the attending physician and the number of packs actually purchased in pharmacies were analyzed in TO and TI by means of Electronic Medicines Prescriptions (PEM) records for the following drugs: bronchodilators (BD), inhaled corticosteroids isolated or in combination with bronchodilators (OUT), oral antihistamines (AH), leukotriene antagonists (LCRA), nasal corticosteroids (CN) and oral corticosteroids (CO). The following demographic and clinical variables were analyzed: age, sex, clinical diagnosis, atopy, allergen sensitization and specific immunotherapy treatment (ITE). Results: We found a compliance of $64.76 \%$ to the prescription. The drugs which the patients most adhere were oral corticosteroids (73\%), followed by leukotriene antagonists and antihistamines (70\%). When analysing associations between variables, it was observed that patients who were not under ITE had greater adhesion to the inhalers (BD and $C I)(p<0.05)$. The asthmatic group had a positive association with adherence to the LCRA $(p<0.05)$ and in the analysis by age, we found that the infant population had a positive association with adherence to $\mathrm{AH}(p<0.05)$. Conclusions: To improve adherence to therapy in asthma, it is important to address and know patient's compliance. The study of each patient's adherence based on computerized drug prescription and retrieval systems in pharmacies allows prescribing physicians to introduce this variable into the analysis of asthma control.

Keywords: Asthma, adherence, electronic prescription, medication use.

\section{INTRODUÇÃO}

A asma brônquica é uma doença crónica que afeta cerca de 235 milhões de pessoas a nível mundial'. Na Europa cerca de 30 milhões de crianças e adultos com idade inferior a 45 anos têm asma brônquica $^{2}$ e em Portugal estima-se que esta doença afete cerca de $6,8 \%$ da população ${ }^{3}$.

Esta patologia tem um impacto económico significativo, quer em termos de custos diretos (consultas médicas, idas ao serviço de urgência, internamentos hospitalares, medicamentos, exames auxiliares de diagnóstico), quer indiretos (ausências laborais) ${ }^{4}$. Estudos mostram que os custos dos cuidados de saúde aumentam no caso dos doentes asmáticos com a má adesão à terapêutica, condicionando o mau controlo da doença um maior recurso aos serviços de urgência e absentismo laboral ${ }^{5}$. A adesão à terapêutica de controlo em crianças e adultos asmáticos varia entre 30 a $70 \%{ }^{6}$, pelo que a otimização da adesão à prescrição reduziria o custo geral dos cuidados de saúde nestes doentes, bem como o controlo da sua doença e qualidade de vida associada ${ }^{7}$.

A adesão à terapêutica é definida pela Organização Mundial de Saúde (OMS) como o grau de correspondência entre o comportamento de uma pessoa em relação às re- 
comendações efetuadas pelos profissionais de saúde, nomeadamente no que refere à medicação prescrita, ao cumprimento de dieta ou a mudanças no seu estilo de vida ${ }^{8}$.

A não adesão pode ser primária no sentido em que o doente não consegue renovar a receita/prescrição por dificuldades no acesso aos cuidados de saúde, ou secundária pelo incumprimento da terapêutica proposta pela alteração voluntária das doses e/ou duração de tratamento?.

A não adesão secundária pode dever-se a fatores relacionados ou não com a medicação. Nos fatores relacionados com a medicação incluem-se a não compreensão da necessidade dos fármacos de controlo ou de SOS, o plano de tratamento pouco prático (muitas vezes/dia ou múltiplos fármacos), dificuldades na utilização dos inaladores, medo dos efeitos secundários, dificuldades económicas, por alguma razão não gostar ou não se adaptar à medicação. Os fatores não relacionados com a medicação incluem não acreditar ou negar a causa dos sintomas ou das crises, não compreender o plano de tratamento, ter expectativas inapropriadas, falta de orientações para o autocontrolo, insatisfação com os profissionais de saúde, medos/ preocupações não expressos ou não discutidos, motivos culturais (como tradições e crenças acerca da asma e do seu tratamento) ${ }^{10}$.

A OMS define ainda três tipos de não adesão: a adesão errática, que consiste numa forma não intencional de não adesão, relacionada com o esquecimento; a não adesão inteligente, em que o doente propositadamente descontinua ou até nem chega a iniciar o tratamento (por exemplo, por se sentir melhor ou por achar que não precisa); e a não adesão não intencional, na qual o doente não compreendeu inteiramente as indicações do plano terapêutico e/ou a necessidade de o cumprir ${ }^{8}$.

No acompanhamento dos doentes asmáticos é essencial que o médico assistente perceba $\circ$ grau de adesão à terapêutica, no sentido de otimizar a prescrição e atingir o controlo da doença. Na prática clínica, o médico pode recorrer a algumas ferramentas, como o autorrelato, questionando diretamente o doente acerca do cumprimento da medicação prescrita. Contudo, os estudos mostram que os doentes tendem a não querer desapontar os mé-

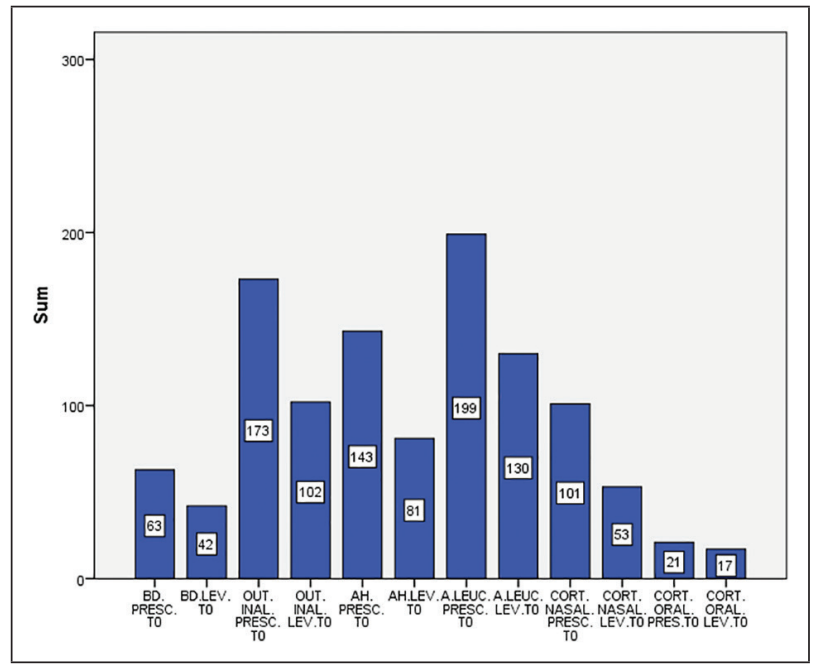

Figura I. Número de embalagens prescritas e levantadas por categoria de medicamento em T0

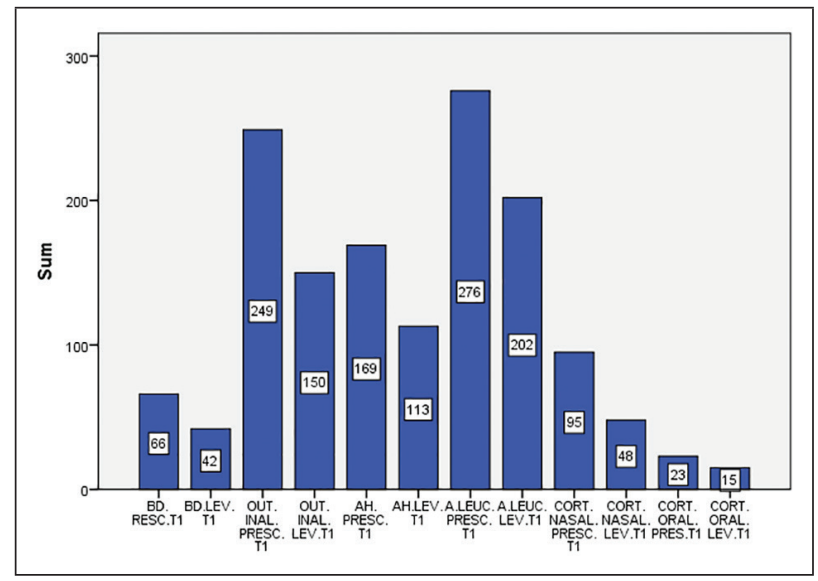

Figura 2. Número de embalagens prescritas e levantadas por categoria de medicamento em TI

dicos, pelo que ocorre uma sobrestimativa do consumo da medicação prescrita". Em alguns países existem monitores eletrónicos aplicados nos dispositivos de medicação inalada. Estes permitem a avaliação do padrão de consumo do doente e a sua relação com os sintomas e apresentam assim registos mais fidedignos da adesão dos doentes. Atendendo aos seus custos, estes monitores são normalmente reservados para os doentes mais graves e que recorrem com mais frequência aos cuidados de saúde. São 
também utilizados em ensaios clínicos no sentido de avaliar o correto cumprimento dos protocolos de investigação ${ }^{12,13}$. Alguns estudos mostram também a utilidade dos questionários preenchidos pelos doentes sobre a sua adesão à medicação, verificando-se que esta ferramenta facilita o autorrelato de não adesão e a identificação de barreiras específicas para cada doente ${ }^{14}$. Alguns dispositivos inalatórios apresentam um contador de doses, o que possibilita um cálculo aproximado da adesão ao medicamento em causa (fazendo a média diária da medicação dispensada pelo inalador no período avaliado). Alguns estudos demonstram vantagens na utilização de dispositivos inalatórios com monitorização eletrónica do seu uso ${ }^{15}$ e mais recentemente têm sido desenvolvidas aplicações para smartphones para monitorização e melhoria de adesão à medicação inalada nos doentes asmáticos ${ }^{16,17}$.

Também a prescrição eletrónica permite atualmente obter informação acerca da aquisição da medicação nas farmácias, sendo uma forma de avaliação da adesão do doente $^{18,19,20,21}$, verificando-se mesmo que esta modalidade de prescrição, em comparação com as receitas em papel, parece aumentar a adesão dos doentes ${ }^{22}$.

\section{OBJETIVOS}

Este estudo tem como objetivo avaliar a adesão dos doentes asmáticos à medicação prescrita pela análise dos medicamentos levantados a partir da informação disponibilizada pela plataforma de prescrição médica eletrónica (PEM).

\section{MÉTODOS}

\section{Desenho do estudo}

Trata-se de um estudo retrospetivo com os seguintes critérios de inclusão:

- Doentes com asma brônquica seguidos em consulta de Imunoalergologia do Hospital São Bernardo entre janeiro de 2016 e dezembro de 2017.
Foram selecionados aleatoriamente 63 doentes tendo em conta os critérios de inclusão descritos.

Foi efetuada uma análise retrospetiva dos processos clínicos desses doentes e analisadas as seguintes variáveis demográficas e clínicas: idade, sexo, diagnósticos clínicos (asma / asma e rinite), atopia (referência explícita a doença alérgica no diário clínico ou resultado positivo em pelo menos um teste cutâneo por picada ou resultado positivo a pelo menos uma IgE específica a alergénios), realização de imunoterapia específica (ITE).

Foram estudados dois períodos de I ano: de janeiro a dezembro de 2016 (T0) e de janeiro a dezembro de 2017 (TI) e, para cada doente, com base nos registos de prescrição electrónica de medicamentos (PEM), foi analisado o número de embalagens prescritas pelo médico assistente e o número das embalagens efetivamente adquiridas nas farmácias, relativamente aos seguintes fármacos: broncodilatadores (BD), corticoides inalados ou em associação com broncodilatadores (outros inaladores - OUT), anti-histamínicos orais (AH), antagonistas dos leucotrienos (LCRA), corticoides nasais (CN) e corticoides orais (CO).

\section{Análise estatística}

A análise estatística foi feita utilizando o programa SPSS (IBM ${ }^{\circledR}$ SPSS, Chicago, IL, EUA), versão 20.

Foi realizada análise descritiva dos dados para caracterizar as variáveis em cada momento (T0 e TI) através de frequências absolutas para os resultados média e desvio-padrão. Para estudar associações entre as variáveis (sexo, idade, patologia e ITE) e adesão (diferença entre medicamentos prescritos e adquiridos) para cada classe de fármacos em T0 e TI utilizou-se o teste Waldi-Chi-Square. Foram considerados como significativos valores de $p \leq 0,05$.

\section{RESULTADOS}

Dos 63 doentes incluídos, 38 eram do sexo feminino e 25 do sexo masculino. Apresentavam uma média de 
Quadro I. Variáveis demográficas e clínicas

\begin{tabular}{|l|c|}
\hline \multicolumn{1}{|c|}{ Variáveis } & $\mathbf{N}(\%)$ \\
\hline Sexo & $38(60,3)$ \\
- Feminino & $25(39,7)$ \\
- Masculino & \\
\hline Idade & $31(49,2)$ \\
$<18$ anos & $32(50,8)$ \\
$\geq$ I8 anos & \\
\hline Diagnósticos/patologia & $23(36,5)$ \\
- Asma & $39(61,9)$ \\
- Asma+rinite & $1(1,6)$ \\
- Asma+rinite+eczema & \\
\hline Atopia & $50(79,4)$ \\
- sim & $13(20,6)$ \\
- não & \\
\hline ITE & $21(33,3)$ \\
- sim & $42(66,7)$ \\
\hline - não & \\
\hline
\end{tabular}

Quadro 2. Número total de embalagens prescritas e levantadas e percentagem de adesão à prescrição por categoria de medicamento e por ano

\begin{tabular}{|l|c|c|c|}
\hline \multicolumn{1}{|c|}{ Medicamentos } & Ano I & Ano 2 & $\begin{array}{c}\text { Global } \\
\text { (Anol } \\
\text { +Ano2) }\end{array}$ \\
\hline Broncodilatadores & & & \\
Prescritos (n) & 63 & 66 & 129 \\
Levantados (n) & 42 & 42 & 84 \\
Adesão (\%) & 66,7 & 63,6 & 65,1 \\
\hline Outros inaladores & & & \\
Prescritos (n) & 173 & 249 & 422 \\
Levantados (n) & 102 & 150 & 252 \\
Adesão (\%) & 59 & 60,2 & 59,7 \\
\hline A-HI & & & \\
Prescritos (n) & 143 & 169 & 312 \\
Levantados (n) & 81 & 113 & 189 \\
Adesão (\%) & 56,6 & 66,9 & 69,6 \\
\hline Antagonistas leucotrienos & & & \\
Prescritos (n) & 199 & 276 & 475 \\
Levantados (n) & 130 & 202 & 332 \\
Adesão (\%) & 65,3 & 73,2 & 69,9 \\
\hline Corticoides nasal & & & \\
Prescritos (n) & 101 & 95 & 196 \\
Levantados (n) & 53 & 48 & 101 \\
Adesão (\%) & 52,5 & 50,5 & 51,5 \\
\hline Corticoides orais & & & \\
Prescritos (n) & 17 & 23 & 44 \\
Levantados (n) & 81 & 65,2 & 72,7 \\
Adesão (\%) & & & \\
\hline
\end{tabular}

idades de $28 \pm 21$ anos. Os doentes apresentavam como diagnósticos principais asma e rinite (62\%), seguido de asma isolada (37\%). A maioria dos doentes eram atópicos (79\%) e apenas um terço estava a fazer imunoterapia específica (Quadro I).

Globalmente constatamos uma adesão à prescrição de 64,76\% (Quadro 2). Mais especificamente, a categoria de medicamentos com maior adesão foram os corticoides orais (73\%), seguidos dos antagonistas dos leucotrienos (70\%), dos anti-histamínicos (70\%), dos broncodilatadores (65\%), dos corticoides inalados, isolados ou em associação com broncodilatadores (60\%), e dos corticoides nasais (52\%).

$\mathrm{Na}$ análise das embalagens prescritas por ano estudado, verificamos uma consistência na classe de medicamentos com maior adesão, sendo os corticoides orais os mais adquiridos face ao que foi prescrito. No pólo oposto temos os corticoides tópicos nasais, os menos adquiridos em ambos os anos estudados (Figuras I e 2).

Verificou-se ainda uma maior prescrição de embalagens de todas as classes de medicamentos estudados em TI, com exceção dos corticoides nasais (Figura 3).

$\mathrm{Na}$ análise dos medicamentos adquiridos por grupo etário (criança /adulto), verificamos que se mantêm os cor-

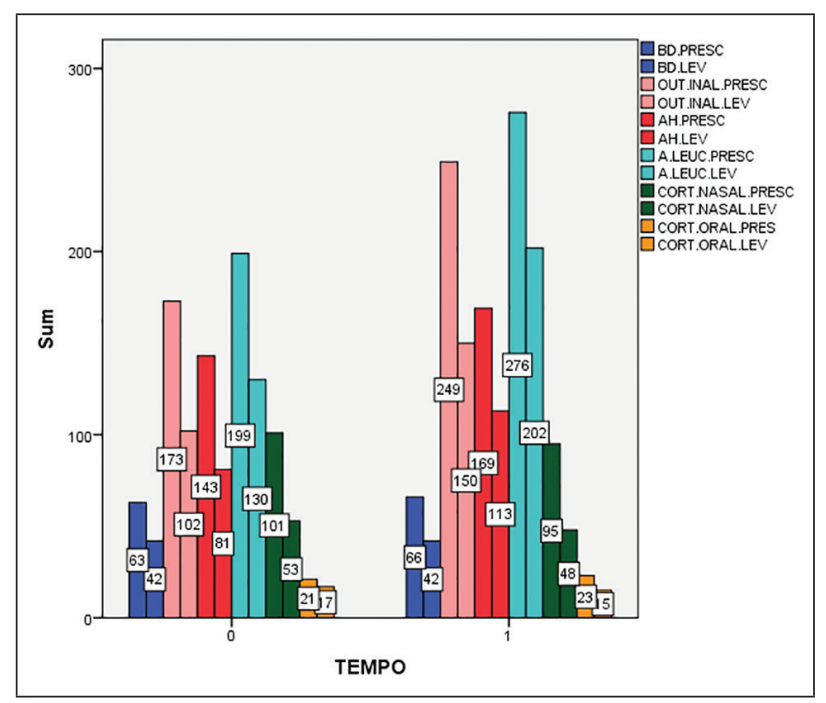

Figura 3. Número de embalagens prescritas e levantadas por categoria de medicamento em T0 e TI 
Marta Martins, Bárbara Cardoso, Sofia Farinha, Rute Reis, Elza Tomaz, Filipe Inácio

Quadro 3. Percentagem de adesão à prescrição por categoria de medicamento na idade adulta e pediátrica

\begin{tabular}{|l|c|c|}
\hline \multicolumn{1}{|c|}{ Medicamentos } & Adultos & Crianças \\
\hline $\begin{array}{l}\text { Broncodilatadores } \\
\text { Adesão (\%) }\end{array}$ & 70,7 & 61,3 \\
\hline $\begin{array}{l}\text { Outros inaladores } \\
\text { Adesão (\%) }\end{array}$ & 57,2 & 64,1 \\
\hline $\begin{array}{l}\text { A-h I } \\
\text { Adesão (\%) }\end{array}$ & 58,7 & 64,6 \\
\hline $\begin{array}{l}\text { Antagonistas leucotrienos } \\
\text { Adesão (\%) }\end{array}$ & 70,6 & 69 \\
\hline $\begin{array}{l}\text { Corticoides nasal } \\
\text { Adesão (\%) }\end{array}$ & 51,7 & 51,45 \\
\hline $\begin{array}{l}\text { Corticoides orais } \\
\text { Adesão (\%) }\end{array}$ & 72,7 & 81,25 \\
\hline
\end{tabular}

ticoides orais como a categoria com maior adesão, seguida dos antagonistas dos leucotrienos. Nas crianças, verifica-se uma maior adesão à medicação de controlo inalada (outros inaladores) do que nos adultos. Os corticoides nasais são a classe de medicamentos menos adquirida pelos doentes, independentemente da idade (Quadro 3).

$\mathrm{Na}$ análise de possíveis associações entre as variáveis consideradas (sexo, idade, patologia, ITE) verificámos em T0 uma associação estatisticamente significativa entre a adesão (diferença entre medicamentos prescritos e adquiridos) na categoria dos broncodilatadores $(p=0,002)$ e dos outros inaladores $(p=0,04 I)$ nos doentes que não estão sob ITE. Verificamos ainda uma associação positiva na adesão aos anti-histamínicos orais na idade pediátrica em T0 $(p=0,019)$.

Quadro 4. Tests of model effects

\begin{tabular}{|l|c|c|c|}
\hline \multicolumn{1}{|c|}{ Variáveis } & Wald-Chi-Square & df & Sig. \\
\hline Sexo & $\mathrm{I}, 810$ & $\mathrm{I}$ & 0,179 \\
\hline Idade & 3,621 & 2 & 0,164 \\
\hline Patologia & 2,252 & $\mathrm{I}$ & 0,133 \\
\hline ITE & 9,593 & $\mathrm{I}$ & 0,002 \\
\hline
\end{tabular}

Variável dependente adesão aos broncodilatadores em T0
Quadro 5. Tests of model effects

\begin{tabular}{|l|c|c|c|}
\hline \multicolumn{1}{|c|}{ Variáveis } & Wald-Chi-Square & df & Sig. \\
\hline Sexo & 0,060 & I & 0,807 \\
\hline Idade & 1,735 & 2 & 0,420 \\
\hline Patologia & 0,934 & I & 0,334 \\
\hline ITE & 4,157 & I & 0,04 I \\
\hline
\end{tabular}

Variável dependente adesão a outros inaladores em T0

Quadro 6. Tests of Model Effects

\begin{tabular}{|l|c|c|c|}
\hline \multicolumn{1}{|c|}{ Variáveis } & Wald-Chi-Square & df & Sig. \\
\hline Sexo & 0,0586 & I & 0,807 \\
\hline Idade $<18$ anos & 5,487 & I & 0,019 \\
\hline Patologia & 0,456 & I & 0,499 \\
\hline ITE & 2,99 I & I & 0,084 \\
\hline
\end{tabular}

Variável dependente adesão a A-hl orais em T0

Quadro 7. Tests of model effects

\begin{tabular}{|l|c|c|c|}
\hline \multicolumn{1}{|c|}{ Variáveis } & Wald-Chi-Square & df & Sig. \\
\hline Sexo & 0,016 & I & 0,898 \\
\hline Idade & 1,307 & 2 & 0,520 \\
\hline $\begin{array}{l}\text { Patologia I } \\
\text {-Asma }\end{array}$ & 4,234 & I & 0,040 \\
\hline ITE & 1,346 & I & 0,246 \\
\hline
\end{tabular}

Variável dependente adesão a antagonistas dos leucotrienos em $\mathrm{TI}$

Em TI observamos uma relação estatisticamente significativa entre a adesão aos antagonistas dos leucotrienos e a asma (não acompanhada de rinite) $(p=0,040)$.

\section{DISCUSSÃo}

A asma é uma doença respiratória crónica com impacto significativo na sociedade, contribuindo para tal a sua importante prevalência e o facto de uma percentagem 
significativa dos doentes asmáticos não terem a sua doença controlada ${ }^{23,24}$. A adesão ao tratamento instituído é um fator determinante para o controlo da asma; no entanto, esta é uma das doenças crónicas com taxas mais baixas de adesão ao tratamento. Alguns estudos reportam que apenas 8 a 13\% dos asmáticos que adquirem inicialmente o corticoide inalado continuam a fazer o reabastecimento do medicamento, fazendo mesmo assim menos de metade das doses prescritas 25,26 .

Os estudos sobre a adesão à terapêutica na asma têm recorrido a diferentes metodologias, nomeadamente à informação de base de dados das farmácias. Alguns destes estudos concluíram que apenas cerca de $28 \%$ das prescrições de medicamentos para a asma e para a doença pulmonar crónica tinham tido uma adesão satisfatória ${ }^{20,21}$, nomeadamente no que refere aos corticoides inalados ${ }^{21}$.

Num outro estudo que incluiu 37 I adultos asmáticos e que avaliou a adesão através de entrevista aos doentes, verificou-se que apenas $26 \%$ dos doentes usou a medicação de controlo de forma adequada ${ }^{27}$.

No presente estudo, a percentagem de adesão à terapêutica de controlo da asma, nomeadamente a corticóides inalados isolados ou em associação com broncodilatadores, foi de $59,72 \%$, o que está de acordo com estudos anteriores que reportam uma adesão à corticoterapia inalada entre os 30 a $70 \%{ }^{28}$. Ainda no que se refere à medicação de controlo, verificamos uma adesão de $69,89 \%$ nos antagonistas dos leucotrienos e que os doentes asmáticos que não apresentavam rinite mostraram maior adesão a esta categoria de medicamentos. Outros estudos têm observado uma maior da adesão aos LCRA versus $\mathrm{Cl}^{29,30,31}$. Alguns autores apontam como possível explicação o facto dos doentes aceitarem melhor regimes terapêuticos mais simples (comprimido versus inalador) ${ }^{32}$.

Neste estudo verificou-se uma adesão global nas crianças de $64,3 \%$; outros estudos têm também constatado que apenas cerca de $50 \%$ das crianças asmáticas aderem à medicação prescrita ${ }^{33,34}$.

Verificamos ainda uma associação positiva entre a adesão aos anti-histamínicos orais e a idade pediátrica,
- que poderá estar relacionado eventualmente com a perceção de segurança desta classe de medicamentos e à observação de alívio mais rápido dos sintomas alérgicos pelos pais e cuidadores. Para além dos ensaios clínicos, que não reproduzem as condições de vida real, os estudos sobre a adesão aos anti-histamínicos são escassos. Num estudo que incluiu adultos e crianças com idade acima de 12 anos, observou-se uma adesão de cerca de $80 \%$ a esta categoria de fármacos, avaliada através de questionários aos médicos e doentes ${ }^{35}$, resultados superiores aos encontrados no estudo atual (70\%), o que poderá dever-se à diferença de metodologia utilizada na avaliação da adesão.

\section{CONCLUSÕES}

Não existe um método de avaliação da adesão à terapêutica que possa ser considerado gold standard, pelo que os métodos disponíveis permitem apenas uma estimativa do comportamento de adesão do doente. Contudo, esta análise parece ser essencial, principalmente no que se refere a otimização do controlo das doenças crónicas, como são exemplo as doenças alérgicas.

A análise da adesão de cada doente com base nos sistemas informáticos de prescrição através do registo de aquisição dos medicamentos nas farmácias, como são exemplo a PEM, ainda que não sejam 100\% fidedignas, até por falhas dos próprios sistemas, permitem aos médicos prescritores introduzir esta variável na análise do controlo da doença. De salientar que esta análise não tem em conta o número de doses/tomas administradas das embalagens realmente adquiridas, pelo que não deixa de ser uma avaliação aproximada da real adesão dos doentes. De qualquer forma poderá ser uma ferramenta útil para avaliar e indagar os doentes acerca de potenciais barreiras à adesão e promover, se necessário, o ajuste terapêutico (nomeadamente regimes, doses, formulações, dispositivos inalatórios), e assim otimizar o controlo da doença. 


\section{Conflito de interesses}

Os autores declaram que não existem conflitos de interesse.

\section{Contacto:}

Marta Isabel Pereira Martins Batista

Serviço de Imunoalergologia

Hospital São Bernardo

R. Camilo Castelo Branco

2910-446 Setúbal

marttamarttins@gmail.com

\section{REFERÊNCIAS}

I. World Health Organization. Asthma, 2017. Available from: http:// www.who.int/en/news-room/fact-sheets/detail/asthma

2. Gibson GJ, Loddenkemper R, Lundback Bo. The European Lung White Book: Respiratory Health and Disease in Europe (2nd ed). European Respiratory Society, Sheffield, 2013. Available from: www.erswhitebook.org

3. Sa-Sousa A, Morais-Almeida M, Azevedo LF, Carvalho R, Jacinto T, Todo-Bom A, et al. Prevalence of asthma in Portugal - The Portuguese National Asthma Survey. Clinical and translational allergy 2012; 2:I-I5.

4. Nunes, C.; Ladeira, S. O impacto económico da asma em avaliação contínua. Rev Port Imunoalergologia 2004;12:1/4-28.

5. Murphy KR, Meltzer EO, Blaiss MS, Nathan RA, Stoloff SW, and Doherty DE: Asthma management and control in the United States: results of the 2009 Asthma Insight and Management survey. Allergy Asthma Proc 2012; 33: 54-64.

6. Eakin M. N., and Rand C.S. Improving patient adherence with asthma self-management practices: what works? Ann Allergy Asthma Immunol 2012; 109:90-2.

7. Schlender A, Alperin PE, Grossman HL, and Sutherland ER. Modeling the impact of increased adherence to asthma therapy. PloS One 2012; 7: p e5II39

8. World Health Organization. Adherence to long-term therapies: evidence for action. Annex 2003;1:143.

9. Weinstein AG. Asthma adherence management for the clinician. J Allergy Clin Immunol Pract 2013;1:123-8.

10. Direcção-Geral da Saúde. Manual de boas práticas na asma. Comissão de Coordenação do Programa Nacional de Controlo da Asma; Revisto em novembro 2007.

II. Milgrom H, Bender B, Ackerson L, Bowry P, Smith B, Rand C. Noncompliance and treatment failure in children with asthma. J Allergy Clin Immunol 1996;98:I05I-7.

12. Bender B, Wamboldt FS, O'Connor SL, Rand C, Szefler S, Milgrom $\mathrm{H}$, et al. Measurement of children's asthma medication adherence by self report, mother report, canister weight, and Doser CT. Ann Allergy Asthma Immunol 2000;85: 416-2I.

13. Patel M, Pilcher J, Travers J, Perrin K, Shaw D, Black P, et al. Use of metered dose inhaler electronic monitoring in a real-world asthma randomized controlled trial. J Allergy Clin Immunol: In Practice 2013;1:83-9|.

14. Schatz M, Zeiger RS, Yang S-J, Weinstein AG, Chen W, Saris-Baglama RN, et al. Development and preliminary validation of the adult asthma adherence questionnaire TM. J Allergy Clin Immunol: In Practice 2013;1:280-8.

15. Chan AH, Steward AW, Harrison J, Camargo CA Jr, Black PN, Mitchell EA. The effect of an electronic monitoring device with audiovisual reminder function on adherence to inhaled corticosteroids and school attendance in children with asthma: a randomized controlled trial. The Lancet Respiratory Medicine 2015;3:210-9.

16. Marcano Belisario J.S., Huckvale K, Greenfield G, Carl J, Gunn LH. Smartphone and tablet self management apps for asthma. The Cochrane database of systematic reviews 2013:CD010013.

17. Jácome C, Guedes R, Almeida R, Teixeira JF, Pinho B, Vieira-Marques P, et al. mINSPIRERS - Estudo da exequibilidade de uma aplicação móvel para medição e melhoria da adesão à medicação inalada de controlo em adolescentes e adultos com asma persistente - Protocolo de um estudo observacional multicêntrico. Rev Port Imunoalergologia 2018;26(I):47-6I.

18. Vink NM, Klungel OH, Stolk RP, Denig P. Comparison of various measures for assessing medication refill adherence using prescription data. Pharmacoepidemiol Drug Saf 2009;18:159-65.

19. Weinstein A.G. Improving adherence to asthma therapies. Curr Opin Pulm Med 2015;21:86-94.

20. Krigsman K, Nilsson JLG, Ring L. Refill adherence for patients with asthma and COPD: comparison of a pharmacy record database with manually collected repeat prescriptions. Pharmacoepidemiology and Drug Safety 2007;16:44I-8.

21. Krigsman K., Moen J., Nilsson JLG, Ring L. Refill adherence by the elderly for asthma/chronic obstructive pulmonary disease drugs dispensed over a 10-year period. Journal of Clinical Pharmacy and Therapeutics 2007; 32:603-II.

22. Surescripts. The national progress report on e-prescribing and interoperable health care 20II. Available at http:// www.surescripts.com

23. Demoly P, Paggiaro P, Plaza V, Bolge SC, Kannan H, Sohier B, et al. Prevalence of asthma control among adults in France, Germany, Italy, Spain and the UK. European Respiratory Review 2009; I8(II2):105-12.

24. Zahran HS, Bailey CM, Qin X, Johnson C. Long-term control medication use and asthma control status among children and adults with asthma. J Asthma 2017;54(10):1065-72.

25. Borrelli B, Riekert KA, Weinstein A, Rathier L. Brief motivational interviewing as a clinical strategy to promote asthma medication adherence. J Allergy Clin Immunol 2007; 120:1023-30. 
26. Bender BG. Advancing the science of adherence measurement:implications for the clinician. J Allergy Clin Immunol Pract 2013;1:92$-3$.

27. Kandane-Rathnayake RK, Matheson MC, Simpson JA, Tang MLK, Johns DP, Mészáros D, et al. Adherence to asthma management guidelines by middle-aged adults with current asthma. Thorax 2009;64(I2):|025-3|.

28. Bender B, Milgrom H, Rand C. Nonadherence in asthmatic patients: Is there a solution to the problem? Annals of allergy, Asthma \& Immunology 1997; 79(3):I77-87.

29. Jones C, Santanello NC, Boccuzzi SJ, Wogen J, Strub P, Nelsen LM. Adherence to Prescribed Treatment for Asthma: Evidence from Pharmacy Benefits Data. Journal of Asthma 2003; 40(I): 93-10I.

30. Mendelsohn AB, Tomaszewski EL, Shah AJ. Treatment adherence in patients with asthma taking leukotriene modifiers versus those taking inhaled corticosteroids. Value in Health 2014; 17(3): Al78
31. Drazen JM, Boccuzzi SJ, Wogen J, Strub P, Jones C, Nelson L, et al. Adherence to prescribed treatment for asthma [abstract]. Am J Respir Crit Care Med 2000;161:A402.

32. Bender BG. Overcoming barriers to nonadherence in asthma treatment. J Allergy Clin Immunol 2002;109(6 Suppl):S554-9.

33. Bender B, Wamboldt FS, O'Conell SL, Rand C, Szefler S, Milgrom $\mathrm{H}$, et al. Measurement of children's asthma medication adherence by self report, mother report, canister weight, and Doser CT. Ann Allergy Asthma Immunol 2000;85:416-2I.

34. Creer TL. Medication compliance and childhood asthma. In: Krasneger NA, Epstein L, Johnson SB, Yaffe SJ, editors. Developmental aspects of health compliance behavior. Hillsdale, NJ: Lawrence Erbaum Associates 1993.p.303-33.

35. Valero A, de la Torre F, CastilloJA, Rivas P, del Cuvillo A, Antépara I, et al. Safety of rupatadine administered over a period of I year in the treatment of persistent allergic rhinitis: a multicentre, open-label study in Spain. Drug Saf 2009; 32:33-42. 